\title{
Evaluación del desarrollo y la estabilidad de una nanoemulsión de vainilla (Vanilla planifolia) a partir de un extracto no alcohólico
}

\author{
Evaluation of the development and stability of a vanilla (Vanilla planifolia) emulsion from a \\ non-alcoholic extract
}

\begin{abstract}
Liliana G. Mendoza Sánchez, Melissa Rodríguez España, Javier Martínez Rodríguez, Hugo S. García Galindo*, Javier De la Cruz Medina, Cynthia Cano Sarmiento y José A. Monroy Rivera†

UNIDA, Tecnológico Nacional de México/Instituto Tecnológico de Veracruz, M.A. de Quevedo 2779, Col. Formando Hogar, Veracruz, Ver. 91897
\end{abstract}

\section{RESUMEN}

La vainilla (Vainilla planifolia) es considerada como el saborizante de mayor importancia en el mundo. Sin embargo, su aplicación en alimentos está limitada, ya que se comercializa principalmente como extractos etanólicos, debido a su naturaleza lipofílica. El objetivo de este trabajo fue elaborar nanoemulsiones de vainilla libre de alcohol, a partir de un extracto natural mediante homogenización por altas presiones, como estrategia para mejorar su solubilidad y estabilidad. El efecto de la presión y el número de ciclos se evaluaron mediante un diseño factorial de $2^{3 *} 3$. Las emulsiones óptimas se almacenaron a 15,25 y $35^{\circ} \mathrm{C}$ durante 42 días. A las emulsiones obtenidas se les evaluó tamaño de partícula e índice de estabilidad (TSI). La formulación óptima para generar una emulsión estable sin azúcar requirió 5 ciclos a una presión de $40 \mathrm{MPa}$, mientras que para la emulsión con azúcar fueron necesarios 9 ciclos a una presión de $20 \mathrm{Mpa}$, con un contenido de $17 \%$ de glicerol. La emulsión se mantuvo estable a 15 y $25^{\circ} \mathrm{C}$ durante 42 días. Los hallazgos de este estudio conducirán a la posibilidad de que esta formulación se utilice como uno de los métodos para mejorar su solubilidad y estabilidad.

Palabras clave: Vainilla planifolia, emulsión, alta presión, estabilidad.

\section{ABSTRACT}

Vanilla (Vanilla planifolia) is considered the most important flavoring in the world. However, its application in foods is limited, since it is mainly commercialized as ethanolic extracts, due to its lipophilic naturalization. The objective of this work was to elaborate alcohol-free vanilla nanoemulsions, from a natural extract by means of high pressure homogenization, as a strategy to improve its solubility and stability. The effects of pressure and number of cycles were evaluated by means of a $2^{3 *} 3$ factorial design. The optimal emulsions were stored at 15,25 and $35^{\circ} \mathrm{C}$ for 42 days. The obtained emulsions were evaluated for particle size and stability index (TSI). The optimum formulation to prepare a stable emulsion without sugar required 5 cycles at a pressure of $40 \mathrm{MPa}$, while for the emulsion with sugar, 9 cycles were necessary at a pressure of $20 \mathrm{MPa}$; with a content of $17 \%$ glycerol. The emulsion remained stable at 15 and $25^{\circ} \mathrm{C}$ for
42 days. The findings of this study will lead to the possibility for the use of this formulation as a method to improve its solubility and stability.

Keywords: Vanilla planifolia, emulsion, high pressure, stability.

\section{INTRODUCCIÓN}

La vainilla es considerada como uno de los saborizantes más populares del mundo; es obtenida principalmente de Vanilla planifolia, una especie de orquídea trepadora tropical. México es el centro de origen y su domesticación, pero actualmente se cultiva en muchos países (Gonzalez-Arnao et al., 2009). La vainillina natural se extrae de las vainas de vainilla mediante un proceso de extracción de alcohol etílico adecuado. La FDA definió un extracto de vainilla como una solución que contiene no menos de $35 \%$ de alcohol etílico extraído de 13.35 oz de vainas de vainilla y que contiene más de $25 \%$ de humedad en un galón de producto terminado, y sin adición de vainillina sintética (Waliszewski et al., 2007).

El sabor y aroma característico del extracto de vainilla es desarrollado en las vainas debidamente curadas, y es el resultado de una serie de transformaciones bioquímicas, donde se han identificado más de 200 componentes, siendo el ácido $p$-hidroxibenzoico, el $p$-hidroxibenzaldehído y el ácido vainillico los principales compuestos que le otorgan el sabor y el aroma característico a vainilla (Sostaric et al., 2000; Sinha et al., 2007; Khoyratty et al., 2018).

Se usa regularmente en la industria artesanal, tabaco, cosméticos, farmacéutica, de alimentos y bebidas (Sinha et al., 2007). Sin embargo, debido a su naturaleza moderadamente lipofílica, generalmente es utilizada como extracto etanólico, lo que limita su uso dentro de la industria alimentaria. El uso de emulsiones, es una estrategia tecnológica que permite mejorar la solubilidad en agua y reducir la degradación o disminución de aroma, sabor y propiedades fisicoquímicas, nutricionales y funcionales durante el procesamiento y almacenamiento, proporcionando durabilidad y protección frente a factores externos como calor, luz y humedad (Jafari et al., 2017).

Las emulsiones de acuerdo con su tamaño de partícula y propiedades se pueden clasificar en macroemulsiones de diámetros de gota mayores a $1 \mu \mathrm{m}$, microemulsiones de 
diámetros de gota en el rango entre 10 y $50 \mathrm{~nm}$, y nanoemulsiones con un tamaño de gota típicamente entre $20 \mathrm{~nm}$ y 500 nm (Yalçinöz y Erçelebi, 2018).

La formulación de nanoemulsiones consiste en mezclar al menos tres componentes, siendo fase oleosa y acuosa más un estabilizador. En general, las nanoemulsiones se pueden preparar utilizando métodos de alta o baja energía (Anton et al., 2008). Los métodos de baja energía se basan en la formación espontánea de diminutas gotas de aceite dentro de sistemas mixtos de aceite, agua y emulsionantes cuando se alteran la solución o las condiciones ambientales, como la composición o la temperatura (Qian y McClements, 2011). Mientras que, los métodos de alta energía, consisten en aplicar altas fuerzas disruptivas con dispositivos mecánicos, como homogeneizadores de alta presión o sonificadores, capaces de provocar la rotura de las gotas de aceite y dispersarlas en la fase acuosa (Tadros et al., 2004).

La estabilidad de las nanoemulsiones se puede mejorar incorporando estabilizadores como tensoactivos de moléculas pequeñas (Tweens o Spans), polisacáridos (goma arábiga o almidón modificado), fosfolípidos (lecitina de soya, huevo o lácteos) y proteínas anfíílicas (caseinatos o aislados de proteína de suero); también se pueden utilizar modificadores de textura, empleando sustancias que aumentan la viscosidad como proteínas (aislados de proteína de suero, gelatina o aislados de proteína de soya), polisacáridos (carragenanos, xantana, pectina, alginato), polioles (sorbitol o glicerol) y azúcares (jarabe de maíz con alto contenido de fructosa o sacarosa) (Salem y Ezzat, 2009).

No obstante, el diseño exitoso y su estabilidad, dependen de una serie de factores, como la selección adecuada de los componentes de la misma y el método de procesamiento. (Kralova y Sjöblom, 2009). Por lo que el objetivo de este estudio fue optimizar la formulación y el proceso de emulsificación de vainilla generadas por altas presiones a partir de un extracto no alcohólico, y estudiar su estabilidad.

\section{MATERIALES Y MÉTODOS}

Todos los compuestos químicos y solventes utilizados fueron grado analítico o HPLC: ácido acético glacial (Baker), Metanol (Burdick y Jackson'), Agua (Burdick y Jackson'), Etanol (Merck) y Glicerol (Golden Bell). Los estándares de vainillina, ácido vainíllico, $p$-hidroxibenzaldehido y ácido $p$-hidroxibenzoico, fueron obtenidos de Sigma (Sigma Chemicals, St. Louis, MO). Los emulsificantes empleados fueron lecitina de Soya (Pronot ultra), Tween 20 (Sigma) y Goma Guar (Sinergum).

\section{Diseño experimental}

El estudio se llevó a cabo en dos etapas. En la primera etapa, se evaluó el efecto de la formulación (Tabla 1), y procesamiento sobre la estabilidad de las emulsiones durante 7 días. En la segunda etapa, las emulsiones óptimas fueron almacenadas a temperaturas controladas, 15,25 y $35^{\circ} \mathrm{C}$, durante 42 días, para estudiar el efecto de la temperatura sobre la estabilidad de la emulsión.

Para desarrollar una formulación óptima de emulsiones de vainilla preparadas por altas presiones, se realizó un diseño de experimentos $2^{3 *} 3$, por lo que en la primera etapa se evaluó el concentrado de vainilla con y sin azúcar (dos variables), presión, número de ciclos y concentración de glicerol (3 niveles) * 3 temperaturas de almacenamiento. En la Tabla 2 , se muestra el tratamiento que recibió cada una de las 24 emulsiones, evaluando la concentración de glicerol, presión y el número de ciclos en las emulsiones con y sin azúcar.

\section{Concentrado de vainilla}

Para eliminar el etanol presente en el extracto de vainilla, se realizó un proceso de evaporación a un lote de 60 $L$ de extracto alcohólico de vainilla con un equipo rotavapor (BÜCHI R-210, Büchi Corp., New Castle, DE), ejerciendo una evaporación al vacío en un rango de 7 a $2.5 \mathrm{kPa}$ y temperatura constante de $25^{\circ} \mathrm{C}$, procesando $1 \mathrm{~L} / \mathrm{h}$ de extracto de vainilla con alcohol.

\section{Análisis cromatográfico}

Se efectuó la cuantificación de los componentes químicos presentes en el extracto alcohólico de vainilla por HPLC, de acuerdo con lo descrito en el Método Oficial de la AOAC 990.25 (AOAC, 1996), con algunas modificaciones. Las separaciones de cromatografías de HPLC se realizaron en un equipo Waters (Milford, MA) a un flujo de $1 \mathrm{~mL} / \mathrm{min}$, usando una bomba binaria Waters 1525, detección a $254 \mathrm{~nm}$ con un detector UV-visible Waters 2487. Se inyectaron $10 \mu \mathrm{L}$ de muestra mediante un auto-muestreador Waters 717 plus. La

Tabla 1. Formulación de las emulsiones de vainilla con y sin azúcar. Table 1. Formulation of vanilla emulsions with and without sugar.

\begin{tabular}{ccccccccc}
\hline & \multicolumn{9}{c}{ Composición (\%) } \\
\cline { 2 - 8 } Formulación & Concentrado de Vainilla & Lecitina & Tween 20 & Goma guar & Glicerol & Azúcar & Agua \\
\hline A & 33.5 & 0.5 & 1.0 & 0.25 & 3.5 & 5 & 56.25 \\
B & 33.5 & 0.5 & 1.0 & 0.25 & 3.5 & - & 61.25 \\
C & 33.5 & 0.5 & 1.0 & 0.25 & 7 & 5 & 52.75 \\
D & 33.5 & 0.5 & 1.0 & 0.25 & 7 & - & 57.75 \\
E & 33.5 & 0.5 & 1.0 & 0.25 & 10 & 5 & 49.75 \\
F & 33.5 & 0.5 & 1.0 & 0.25 & 10 & - \\
\hline
\end{tabular}


Tabla 2. Diseño experimental para la preparación de emulsiones a partir de concentrado de vainilla con y sin azúcar.

Table 2. Experimental design for the preparation of emulsions from vanilla concentrate with and without sugar.

\begin{tabular}{|c|c|c|c|c|}
\hline \multirow[b]{2}{*}{ Emulsión } & \multirow[b]{2}{*}{ Formulación } & \multirow{2}{*}{$\begin{array}{c}\text { Presión } \\
\text { (MPa) }\end{array}$} & \multirow{2}{*}{ Ciclos } & \multirow{2}{*}{$\begin{array}{c}\text { Glicerol } \\
\text { (\%) }\end{array}$} \\
\hline & & & & \\
\hline 1 & A & 20 & 5 & 3.5 \\
\hline 2 & C & 40 & 5 & 7 \\
\hline 3 & $E$ & 20 & 5 & 10 \\
\hline 4 & A & 40 & 5 & 3.5 \\
\hline 5 & C & 20 & 5 & 7 \\
\hline 6 & $\mathrm{E}$ & 40 & 5 & 10 \\
\hline 7 & A & 20 & 9 & 3.5 \\
\hline 8 & C & 20 & 9 & 7 \\
\hline 9 & $\mathrm{E}$ & 20 & 9 & 10 \\
\hline 10 & A & 40 & 9 & 3.5 \\
\hline 11 & C & 40 & 9 & 7 \\
\hline 12 & $E$ & 40 & 9 & 10 \\
\hline 13 & B & 20 & 5 & 3.5 \\
\hline 14 & D & 40 & 5 & 7 \\
\hline 15 & $\mathrm{~F}$ & 20 & 5 & 10 \\
\hline 16 & B & 40 & 5 & 3.5 \\
\hline 17 & D & 20 & 5 & 7 \\
\hline 18 & $\mathrm{~F}$ & 40 & 5 & 10 \\
\hline 19 & B & 20 & 9 & 3.5 \\
\hline 20 & D & 20 & 9 & 7 \\
\hline 21 & $\mathrm{~F}$ & 20 & 9 & 10 \\
\hline 22 & B & 40 & 9 & 3.5 \\
\hline 23 & D & 40 & 9 & 7 \\
\hline 24 & $\mathrm{~F}$ & 40 & 9 & 10 \\
\hline
\end{tabular}

fase móvil se formó por gradiente con $800 \mathrm{~mL}$ agua con $1 \mathrm{~mL}$ ácido acético en la bomba By $95 \%$ de metanol en la bomba $\mathrm{A}$. El programa comienza con $100 \%$ de B y pasa a $95 \%$ de $A$ en $35 \mathrm{~min}$; se mantiene por 5 min más y se regresa a la condición inicial. Se utilizó una columna de fase reversa C18 Dionex (Sunnyvale, CA) de $4.6 \mathrm{~mm} \times 150 \mathrm{~mm}$, con $5 \mu \mathrm{m}$ de tamaño de partícula. Previo a la inyección, se realizó una dilución 1:10 de la muestra en etanol al $99.9 \%$ en un matraz aforado de $10 \mathrm{~mL}$, y fue filtrada a través de microfiltros de membrana de $0.45 \mu \mathrm{m}$ de polifluoruro de vinilo (PVDF, Millipore Corp., Bellington, MA).

\section{Preparación de las emulsiones}

Siguiendo la metodología de Monroy (2013), con algunas modificaciones, se prepararon pre-emulsiones, con azúcar (5\%) y sin azúcar, con un homogeneizador tipo rotorestator UltraTurrax T-25 (IKA Werke, Stauffen, Alemania) a $15,000 \mathrm{rpm}$ durante $5 \mathrm{~min}$ a $15^{\circ} \mathrm{C}$, mezclando lecitina de soya $(0.5 \%)$, Tween $20(1.0 \%)$, goma guar $(0.25 \%)$, concentrado de vainilla (33.5\%), glicerol (3.5, 7 y $10 \%)$, y agua, de acuerdo con lo descrito en la Tabla 1. La emulsión final se preparó con un homogeneizador de pistón de alta presión APV 1000 Lab Series (Crawley, West Sussex, UK). Las condiciones de trabajo se muestran en la Tabla 2.

\section{Medición del tamaño de partícula}

El tamaño medio de partícula de las emulsiones se determinó de acuerdo con lo descrito por Qian y McClements (2011), con algunas modificaciones, mediante dispersión de luz dinámica (DLS), utilizando un equipo Zetasizer NanoZS90 (Malvern Instruments, Worcestershire, UK) en un ángulo detector fijo de $90^{\circ}$. Para minimizar los múltiples efectos de dispersión, antes de cada medición, las dispersiones se diluyeron usando un factor de dilución de 1:1000. Los resultados se describieron como el diámetro medio acumulado (expresado en $\mathrm{nm}$ ) para el tamaño de partícula.

\section{Estabilidad física}

Para estudiar la estabilidad física de las emulsiones, se llevaron a cabo mediciones de retrodispersión (BS) a diferentes tiempos de almacenamiento, utilizando Turbiscan Lab Expert (Formulaction, Toulouse, Francia) (Santos et al., 2020). Los mecanismos de desestabilización fueron cuantificados por el Turbiscan Stability Index (TSI). Este índice es un valor estadístico y su valor se calcula como la suma de todos los procesos de desestabilización en la celda de medición y está definido por:

$$
T S I=\sum_{j}\left|\operatorname{scan}_{r e f}\left(h_{j}\right)-\operatorname{scan}_{i}\left(h_{j}\right)\right|
$$

donde $\operatorname{scan}_{\text {ref }}$ y scan, son el valor de transmisión inicial y el valor de transmisión en un momento específico, respectivamente, y $h_{j}$ es una altura específica en la celda de medición.

\section{Estabilidad de almacenamiento}

La estabilidad de las nanoemulsiones se determinó midiendo el índice de estabilidad (TSI), diariamente durante el almacenamiento a 15,25 y $35^{\circ} \mathrm{C}$ durante 42 días.

\section{Análisis estructural}

Las muestras estudiadas por microscopía electrónica de transmisión fueron preparadas siguiendo el protocolo reportado por Lu et al. (2014), con modificaciones. Se agregaron $10 \mu \mathrm{L}$ de emulsión en una rejilla de cobre cubierta con carbón durante 1 minuto; posteriormente, se hizo una tinción adicionando $10 \mu \mathrm{L}$ de ácido fosfotúngstico a pH 7, secando la muestra a temperatura ambiente durante $1 \mathrm{~h}$. La microestructura de las emulsiones se observó utilizando un microscopio electrónico de trasmisión JEOL TEM-1400 (JEOL, Ltd, Tokyo, Japón), alcanzando hasta 120 kV con una cámara de alta resolución Gatan ES1000W (Pleasantown, CA), de 11 megapixeles y montaje lateral elevado. Las imágenes fueron manejadas en el software de control Digital Micrograph.

\section{Análisis Estadístico}

Todos los experimentos se realizaron por duplicado, y los datos se presentan como promedios \pm desviación es- 
tándar. El análisis estadístico se realizó mediante el software Minitab v. 16.1 (Minitab Inc., State College, PA). Se hizo un análisis de varianza de una vía (one-way ANOVA), usando la prueba Tukey de rangos múltiples para determinar las diferencias significativas entre los tratamientos con un nivel de significancia de $a=0.05$.

\section{RESULTADOS Y DISCUSIÓN}

Análisis cromatográfico

A partir de un extracto etanólico de vainilla y mediante un proceso de rota-evaporación se obtuvieron $15 \mathrm{~L}$ de concentrado de vainilla, el cual fue utilizado para la elaboración de las emulsiones. Los compuestos químicos presentes en el concentrado de vainilla se muestran en la Figura 1. Se identificaron cuatro componentes principales: el ácido $p$ hidroxibenzoico, el $p$-hidroxibenzaldehído, el ácido vainíllico, y la vainillina con tiempos de retención de 26.7, 28.7, 29.3 y 30.6 minutos, respectivamente. Estos compuestos ya han sido previamente identificados y son característicos de $V$. planifolia. Además, son los responsables de proporcionar el sabor y aroma característico (Pérez-Silva et al., 2006; Waliszewski et al., 2007).

La Tabla 3 muestra las concentraciones de dichos compuestos, así como los parámetros de regresión lineal obtenidos para las cuatro curvas de calibración realizadas previamente. El método HPLC utilizado para este estudio logró una adecuada separación entre los cuatro compuestos. La concentración de compuestos en el extracto etanólico de $V$. planifolia osciló entre 0.033 y $0.407 \mathrm{~g} / \mathrm{mL}$. En general, la concentración de vainillina resultó ser la más alta seguida por el ácido vainíllico, el ácido $p$-hidroxibenzoico y el $p$-hidroxibenzaldehído (Tabla 3). Estos datos concuerdan con reportes previos, siendo la concentración del aldehído vainillina la que regularmente se encuentra en concentraciones más altas (Brunschwig et al., 2009; Maruenda et al., 2012; Ferrara, 2020).

\section{Condiciones de procesamiento}

De forma preliminar se elaboró una emulsión de vainilla para determinar las condiciones de presión y número de ciclos, que posteriormente serían evaluadas. La formulación constó de $10 \%$ de concentrado de vainilla, $1 \%$ de Tween $20,0.5 \%$ de lecitina de soya, $0.25 \%$ de goma guar, y $30 \%$ de glicerol. La influencia del número de ciclos y la presión sobre el tamaño de partícula, durante el procesamiento para el desarrollo de emulsiones se muestra en la Figura 2. Para investigar el efecto de la presión de homogeneización sobre el tamaño de partícula, la presión de homogeneización se va-

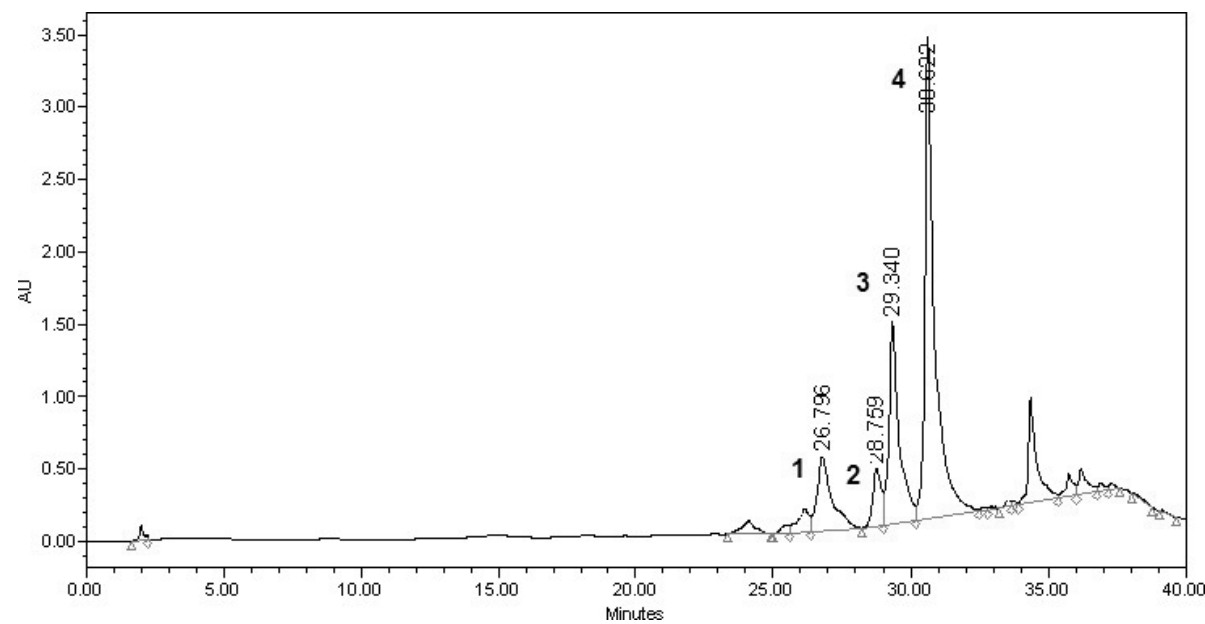

Figura 1. HPLC de fase reversa del extracto de Vanilla planifolia (1) ácido ácido $p$-hidroxibenzoico; (2) p-hidroxibenzaldehido; (3) ácido vainíllico; (4) vainillina.

Figure 1. Reversed-phase HPLC of a Vanilla planifolia extract (1) p-hydroxybenzoic acid; (2) p-hydroxybenzaldehyde; (3) vanillic acid; (4) vanillin.

Tabla 3. Concentración de los compuestos aromáticos encontrados en Vanilla planifolia utilizando HPLC. Table 3. Concentration of the aromatic compounds in Vanilla planifolia using HPLC.

\begin{tabular}{lcccc}
\hline Compuesto & Tiempo de retención & Concentración $\left(\mathbf{g ~ m L}^{-1}\right)$ & \multicolumn{2}{c}{ Regresión lineal } \\
\cline { 4 - 5 } & & & $\mathbf{y}=\mathbf{m} \boldsymbol{x}+\mathbf{b}$ & $\mathbf{r}^{\mathbf{2 a}}$ \\
\hline Ácido $p$-hidroxibenzoico & 26.7 & $0.045 \pm 0.01$ & $5^{-10} \mathbf{x}-6^{-05}$ & 0.9999 \\
$p$-hidroxibenzaldehído & 28.7 & $0.033 \pm 0.04$ & $2^{-10} \mathbf{x}-3^{-4}$ & 0.9983 \\
Ac. Vainíllico & 29.3 & $0.139 \pm 0.01$ & $8^{-10} \mathrm{x}+6^{-4}$ & 0.9988 \\
Vainillina & 30.6 & $0.407 \pm 0.01$ & $3^{-10} \mathrm{x}-1^{-4}$ & 0.9995 \\
\hline
\end{tabular}

a Coeficiente de correlación

Los datos están expresados como la media y \pm error estándar. 


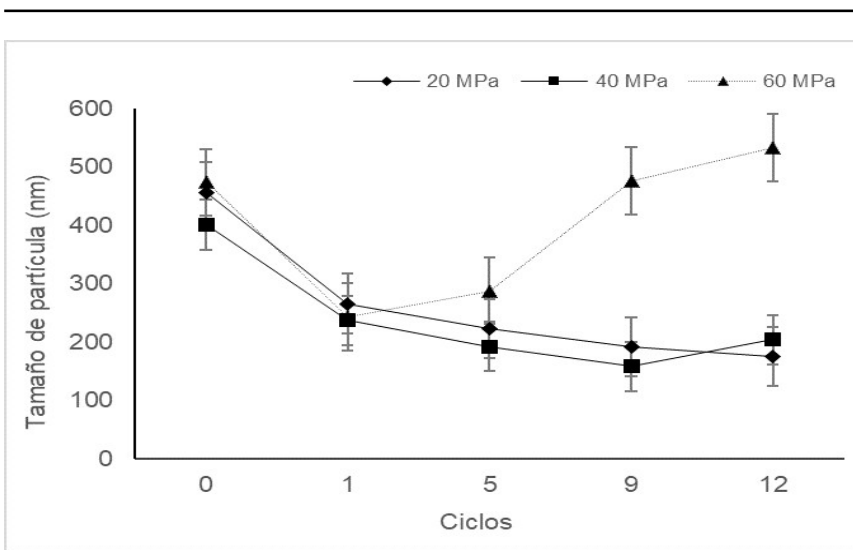

Figura 2. Influencia del número de ciclos sobre el tamaño de partícula en la emulsión de vainilla.

Figure 2. Influence of the number of cycles on the particle size of the vanilla emulsion.

rió de 20 a $60 \mathrm{MPa}$, y el número de ciclos evaluado fue hasta 12. El tamaño de partícula disminuyó cuando la presión aumenta, mostrando comportamientos similares a $20 \mathrm{MPa}$ y 40 $\mathrm{MPa}$. La disminución del tamaño de partícula se debe a que la energía aportada por el proceso de homogeneización fue suficiente para reducir el tamaño de las gotas (Håkansson, 2018). Los tamaños medios de partícula más pequeños a las presiones más grandes son una consecuencia de las tensiones de cizallamiento más altas, representando más energía, mayor turbulencia, e induciendo una tasa de disrupción más alta (Marie et al., 2002; Kotta et al., 2015; Hidajat et al., 2020).

Las emulsiones de vainilla procesadas a $20 \mathrm{MPa}$ con 5 y 9 ciclos mostraron tamaños de partícula de 184.2 y 154 $\mathrm{nm}$, respectivamente, siendo estos los valores de tamaño de partícula más bajos logrados. Sin embargo, se observó un aumento significativo de tamaño de partícula a $60 \mathrm{MPa}$ a partir del ciclo 5, aumentando de 230 hasta $526.7 \mathrm{~nm}$. También, de manera similar se observó un aumento en el tamaño de partícula de la emulsión procesada a $40 \mathrm{MPa}$ en el ciclo 12, mostrando un tamaño de $263 \mathrm{~nm}$. Estos aumentos en el tamaño de partícula a presiones y ciclos más altos, puede deberse al fenómeno denominado sobreprocesamiento o recoalescencia (Qian y McClements, 2011; Hakansson, 2018). Esto se ha informado anteriormente en otros estudios: Ali et al. (2016), obtuvieron tamaños de gotas de hasta $200 \mathrm{~nm}$ de una nanoemulsión estabilizada con $\beta$-lactoglobulina $(\beta-\mathrm{lg})$, cuando se aplicó una presión de homogeneización de 100 $\mathrm{MPa}$, durante 4 ciclos. Sin embargo, la eficiencia de emulsificación de $\beta$-lg se redujo a presiones de homogeneización más altas (200 MPa y $300 \mathrm{MPa}$ ). Por su parte, Santos et al. (2020), desarrollaron emulsiones de aceite esencial de mandarina estabilizado por dos surfactantes de grado alimenticio (Tween 80 y Span 80), y goma guar, y encontraron que los diámetros de las gotas cambiaron hacia tamaños más grandes de uno a dos ciclos, independientemente de la presión de homogeneización, siendo 25000 psi y un ciclo, los que aportaron la energía suficiente para obtener nanoemulsiones de $192 \mathrm{~nm}$ y evitar el fenómeno de sobreprocesamiento.
Existen diversos motivos relacionados con el sobreprocesamiento; los principales están relacionados con la concentración y tasa de adsorción del emulsionante, ya que el área de superficie de las gotas aumenta drásticamente durante la homogeneización a elevadas presiones, y la concentración del emulsionante debería ser suficiente para cubrir toda la interfaz y su tasa de adsorción más alta para evitar la coalescencia, y por lo tanto el aumento en el tamaño de partícula (Jafari et al., 2007).

En este estudio, se encontró que las mejores condiciones de procesamiento para la emulsión de vainilla, de acuerdo con los valores de tamaños de partícula más bajos se encuentran en el rango de los 20 a $40 \mathrm{MPa}$, y de 5 a 9 ciclos, por lo que estas condiciones fueron elegidas para el resto de los experimentos.

\section{Estabilidad física}

Para evaluar la estabilidad de las nanoemulsiones con y sin azúcar se determinó el índice de estabilidad. Las emulsiones fueron preparadas de acuerdo a la formulación y procesamiento que se encuentra descrito en la Tabla 2 . Cada punto en la Figura 3 representa una cinética de 7 días para cada emulsión. Los resultados muestran diferencia significativa entre las emulsiones evaluadas. Se observó una mayor influencia sobre la estabilidad, por el número de ciclos probados y la concentración de glicerol. La estabilidad se vio afectada con la disminución de la concentración de glicerol y el aumento de ciclos en el procesamiento. La emulsión 11 fue la menos estable, mientras que las que mostraron mayor estabilidad fueron las emulsiones 1 y 8 . Sin embargo, la emulsión 1 se descartó para la siguiente evaluación, debido a que exhibió floculación.

El efecto de la formulación y procesamiento en la estabilidad de las emulsiones sin azúcar se observa en la Figura 4. En las emulsiones sin azúcar, la concentración de glicerol ejerció un papel importante en la estabilidad. La emulsión menos estable fue la 19 y las de mayor estabilidad, las 14, 20 y 21. Las emulsiones más estables fueron las formuladas con

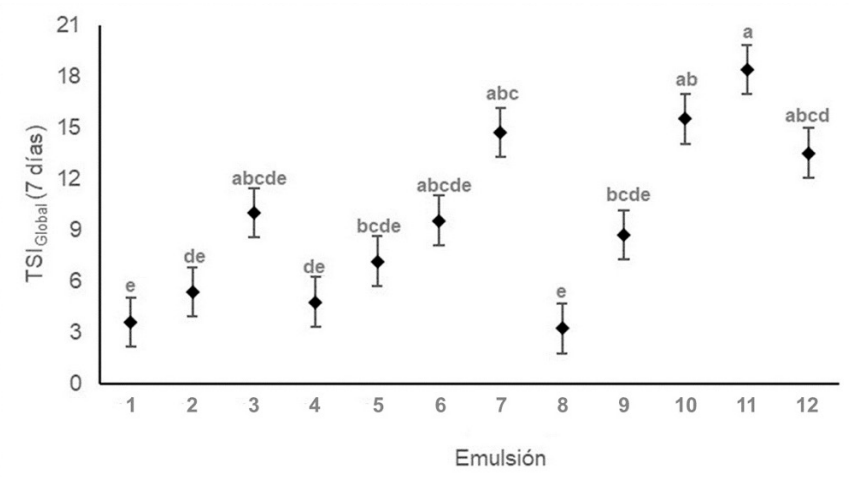

Figura 3. TSI $\mathrm{global}_{\text {al }}$ los días de almacenamiento, en función de las diferentes formulaciones de vainilla con azúcar. Letras diferentes indican diferencias significativas entre las emulsiones a $\mathrm{P}<0.05$

Figure 3. TSI $_{\text {global }}$ at 7 days of storage as a function of the different emulsion formulations of vanilla with sugar. Different letters indicate significant difference between emulsions at $\mathrm{P}<0.05$ 


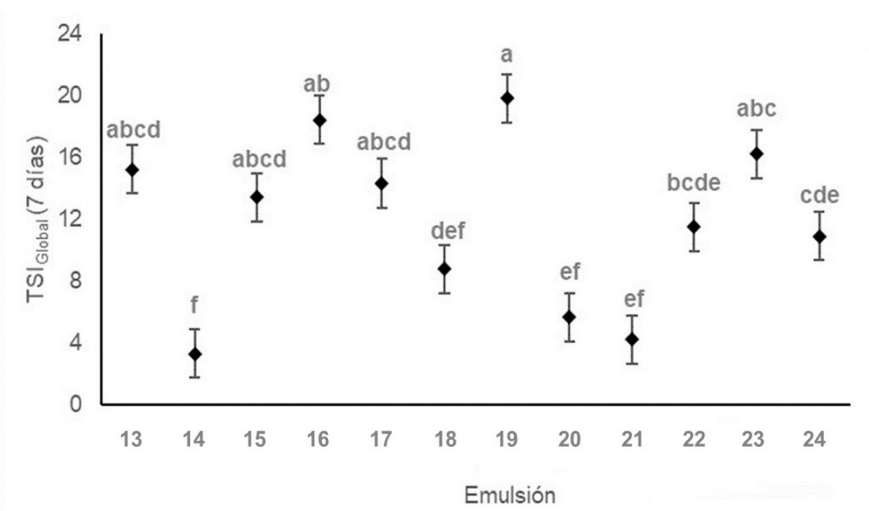

Figura 4. TSI ${ }_{\text {global }}$ a los 7 días de almacenamiento, en función de las diferentes formulaciones de vainilla sin azúcar. Letras diferentes indican diferencias significativas entre las emulsiones a $\mathrm{P}<0.05$

Figure 4. TSI ${ }_{\text {global }}$ at 7 days of storage as a function of the different emulsion formulations of vanilla without sugar. Different letters indicate significant difference between emulsions at $\mathrm{P}<0.05$

mayor concentración de glicerol (7 y $10 \%$ ); esto puede deberse a que al aumentar la concentración de glicerol se produce un cambio en la viscosidad, retardando la difusión de las moléculas del soluto a través de la solución, que promueve la estabilidad; es decir se favorece la formación de sistemas mono-dispersos (McClements, 2004; Chanasattru, 2009). Qian y McClements (2011), modificaron la concentración de glicerol de la fase continua, encontrando una reducción de tamaño al incrementar la concentración de glicerol obteniendo tamaños de hasta $80 \mathrm{~nm}$. Hamed y Abo-Elwafa (2020), reportaron que la viscosidad de la fase continua aumentó al incrementar la concentración de glicerol; sin embargo, este no afectó significativamente las características de la nanoemulsión producida. Tales variaciones en los resultados fueron atribuidas a las diferencias en el tipo de emulsionantes o en el equipo de homogeneización utilizado.

\section{Estabilidad de almacenamiento}

Para investigar el efecto de la temperatura sobre la estabilidad de la emulsión, las emulsiones $8,12,14$ y 23 fueron elegidas como las mejores, y fueron almacenadas a 15,25 y $35^{\circ} \mathrm{C}$, durante 42 días. Los cambios en el índice de estabilidad de las nanoemulsiones de vainilla durante el almacenamiento se muestran en la Figura 5, donde el valor de TSI alto corresponde al sistema más inestable. El índice de estabilidad aumentó durante los 42 días a las tres temperaturas de almacenamiento. Las emulsiones fueron más estables a temperaturas más bajas. Es sabido que la tensión superficial de la mayoría de los líquidos disminuye al aumentar temperatura (Chen y Tao, 2005; Li y Lu, 2016). En sistemas que no están en equilibrio, las emulsiones tienden a reducir sus áreas interfaciales y la energía libre a través de varios procesos de descomposición, como cremado, sedimentación, floculación, maduración de Ostwald y coalescencia (Chantrapornchai et al., 2001; Karthika et al., 2017).

En el presente estudio, la emulsión 8, almacenada a $15^{\circ} \mathrm{C}$ y $25^{\circ} \mathrm{C}$ fue más estable durante los 42 días, mientras que a $35^{\circ} \mathrm{C}$, en el día 21 hubo un incremento de tamaño de

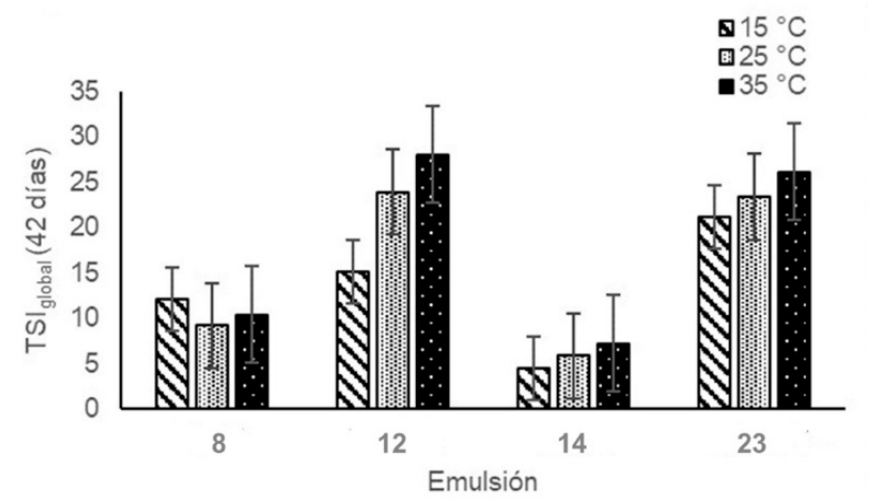

Figura 5. Efecto de la temperatura sobre la TSI ${ }_{\text {global }}$ de las emulsiones con y sin azúcar, durante un almacenamiento de 42 días. Letras diferentes en el gráfico indican diferencia significativa entre temperaturas a $\mathrm{P}<0.05$.

Figure 5. Effect of temperature on the TSI ${ }_{\text {Ilobal }}$ of the emulsions with and without sugar, during a storage of 42 days. Different letters in the graphs indicate significant difference between temperatures at $\mathrm{P}<0.05$.

partícula (datos no mostrados). En la emulsión 14, ocurrió el mismo efecto de inestabilidad que en la emulsión con azúcar a $35^{\circ} \mathrm{C}$, ocurriendo este a los 28 días de almacenamiento; las emulsiones a 15 y $25^{\circ} \mathrm{C}$ no manifestaron algún fenómeno de inestabilidad durante el tiempo de almacenamiento.

\section{Microscopía}

El estudio morfológico de las emulsiones más estables (8 y 14) se realizó mediante microscopía electrónica de transmisión (TEM). Los resultados de TEM permitieron demostrar la formación de las nanoemulsiones y validar los diámetros medios de gota alcanzados. En la emulsión 8 (Figura 6A), se observan estructuras casi esféricas con tamaños de partícula que van de $0.14 \mu \mathrm{m}$ a $0.86 \mu \mathrm{m}$, mientras que para la emulsión 14 (Figura 6B), se observan tamaños de gota de $0.14 \mu \mathrm{m}$ a $0.77 \mu \mathrm{m}$. Estos resultados muestran similitud con los tamaños que fueron alcanzados por la homogeneización de altas presiones y medidos por dispersión de luz dinámica (Figura 2). Dichos valores se encuentran en el rango de 20 a $500 \mathrm{~nm}$, característico de las nanoemulsiones (Ho et al., 2021). En ambas emulsiones se observaron floculos creándose aglomerados; esto indica que probablemente la emulsión tienda a desarrollar sedimentación inversa al pasar los días.

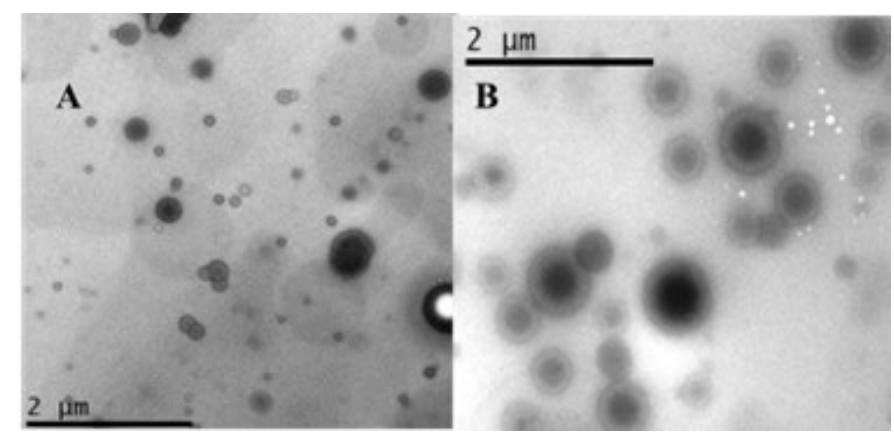

Figura 6. Imágenes de las emulsiones de vainilla obtenidas por un Microscopio electrónico de trasmisión. (A) Emulsión 8; (B) Emulsión 14.

Figure 6. Transmission electron microscopy photographs of vanilla emulsions. (A) Emulsion 8; (B) Emulsion 14. 


\section{CONCLUSIÓN}

El presente estudio confirmó que la estabilidad de la emulsión de vainilla se vio influenciada por la presión de homogeneización y el número de ciclos, así como la formulación de la misma. Se observó que el proceso en un rango de presión entre 20 y $40 \mathrm{MPa}$, es adecuado para evitar el sobreprocesamiento. El incremento en la concentración de glicerol también permitió disminuir el tamaño de partícula y aumentar la viscosidad, contribuyendo con ello en la estabilidad en ambas emulsiones. Una de las observaciones más importantes que se destaca es la estabilidad que le proporcionan el azúcar y el glicerol a las emulsiones, permitiendo extender su tiempo de almacenamiento hasta 42 días con temperaturas menores a $25^{\circ} \mathrm{C}$. Estos hallazgos podrían facilitar su aplicación en diferentes matrices alimentarias, utilizando las nanoemulsiones como una estrategia para preparar ingredientes bioactivos más estables y dispersables en diferentes medios.

\section{AGRADECIMIENTOS}

Los autores agradecen el apoyo del Tecnológico Nacional de México, por el financiamiento bajo el convenio 6229.17-P, y al CONACyT por la beca de estudios de posgrado.

\section{REFERENCIAS}

Ali, A., Mekhloufi, G., Huang, N.y Agnely, F. (2016). $\beta$-lactoglobulin stabilized nanemulsions-Formulation and process factors affecting droplet size and nanoemulsion stability. International Journal of Pharmaceutics, 500(1-2), 291-304. https://doi.org/10.1016/j.ijpharm.2016.01.035

Anton, N., Benoit, J. P. y Saulnier, P. (2008). Design and production of nanoparticles formulated from nano-emulsion templates-A review. Journal of Controlled Release, 128(3), 185-199. https://doi.org/10.1016/j.jconrel.2008.02.007

Brunschwig, C., Collard, F. X., Bianchini, J.-P. y Raharivelomanana, P. (2009). Evaluation of Chemical Variability of Cured Vanilla Beans (Vanilla tahitensis and Vanilla planifolia). Natural Product Communications, 4(10), 1934578X0900401. https:// doi.org/10.1177/1934578X0900401016

Chanasattru, W., Decker, E. A. y McClements, D. J. (2009). Influence of glycerol and sorbitol on thermally induced droplet aggregation in oil-in-water emulsions stabilized by B-lactoglobulin. Food Hydrocolloids, 23(2), 253-261. https:// doi.org/10.1016/j.foodhyd.2008.02.004

Chantrapornchai, W., Clydesdale, F. M. y McClements, D. J. (2001). Influence of relative refractive index on optical properties of emulsions. Food Research International, 34(9), 827-835. https://doi.org/10.1016/S0963-9969(01)00105-3

Chen, G. \& Tao, D. (2005). An experimental study of stability of oil-water emulsion. Fuel Processing Technology, 86(5), 499-508. https://doi.org/10.1016/j.fuproc.2004.03.010

Ferrara, L. (2020). Medicinal and pharmaceutical properties of Vanilla planifolia. International Journal of Medicinal Reviews, 7(1), 25-29. https://doi.org/10.30491 / IJMR.2020.222917.1081

Gonzalez-Arnao, M.T., Lazaro-Vallejo, C. E., Engelmann, F., GamezPastrana, R., Martinez-Ocampo, Y. M., Pastelin-Solano, M. C. y Diaz-Ramos, C. (2009). Multiplication and cryopreservation of vanilla (Vanilla planifolia 'Andrews'). In Vitro Cellular and Developmental Biology - Plant, 45(5), 574-582. https://doi. org/10.1007/s11627-009-9242-6

Håkansson, A. (2018). Fabrication of Nanoemulsions by High-Pressure Valve Homogenization. In S. M. Jafari y D. J. McClements (Eds.), Nanoemulsions (pp. 175-206). Elsevier. https://doi.org/10.1016/B978-0-12-811838-2.00007-2

Hamed, S. F. y Abo-Elwafa, G. A. (2020). Preparation of novel nanoemulsions from omega-3 rich oil. Grasas y Aceites, 71(2), 350. https://doi.org/10.3989/gya.0229191

Hidajat, M. J., Jo, W., Kim, H. y Noh, J. (2020). Effective Droplet Size Reduction and Excellent Stability of Limonene Nanoemulsion Formed by High-Pressure Homogenizer. Colloids and Interfaces, 4(1), 5. https://doi.org/10.3390/ colloids4010005

Ho, T. M., Abik, F. y Mikkonen, K. S. (2021). An overview of nanoemulsion characterization via atomic force microscopy. Critical Reviews in Food Science and Nutrition, 0(0), 1-21. https://doi.org/10.1080/10408398.2021.1879727

Jafari, S. M., He, Y. y Bhandari, B. (2007). Optimization of nanoemulsions production by microfluidization. European Food Research and Technology, 225(5-6), 733-741. https://doi. org/10.1007/s00217-006-0476-9

Jafari, S. M., Paximada, P., Mandala, I., Assadpour, E. y Mehrnia, M. A. (2017). Encapsulation by nanoemulsions. In S. M. Jafari (Ed.), Nanoencapsulation Technologies for the Food and Nutraceutical Industries (pp. 36-73). Elsevier Inc. https://doi. org/10.1016/B978-0-12-809436-5.00002-1

Karthik, P., Ezhilarasi, P. N. y Anandharamakrishnan, C. (2017). Challenges associated in stability of food grade nanoemulsions. Critical Reviews in Food Science and Nutrition, 57(7), 1435-1450. https://doi.org/10.1080/10408398.2015.1 006767

Khoyratty, S., Kodja, H. y Verpoorte, R. (2018). Vanilla flavor production methods: A review. Industrial Crops and Products, 125, 433-442. https://doi.org/10.1016/j.indcrop.2018.09.028

Kotta, S., Khan, A. W., Ansari, S. H., Sharma, R. K. y Ali, J. (2015). Formulation of nanoemulsion: a comparison between phase inversion composition method and high-pressure homogenization method. Drug Delivery, 22(4), 455-466. https://doi.org/10.3109/10717544.2013.866992

Kralova, I. y Sjöblom, J. (2009). Surfactants Used in Food Industry: A Review. Journal of Dispersion Science and Technology, 30(9), 1363-1383. https://doi.org/10.1080/01932690902735561

Li, P.-H. y Lu, W.-C. (2016). Effects of storage conditions on the physical stability of d-limonene nanoemulsion. Food Hydrocolloids, 53, 218-224. https://doi.org/10.1016/j. foodhyd.2015.01.031

Lu, W.-C., Chiang, B.-H., Huang, D.-W. y Li, P.-H. (2014). Skin permeation of d-limonene-based nanoemulsions as a transdermal carrier prepared by ultrasonic emulsification. Ultrasonics Sonochemistry, 21(2), 826-832. https://doi. org/10.1016/j.ultsonch.2013.10.013

Marie, P., Perrier-Cornet, J. M. y Gervais, P. (2002). Influence of major parameters in emulsification mechanisms using a high-pressure jet. Journal of Food Engineering, 53(1), 43-51. https://doi.org/10.1016/S0260-8774(01)00138-8

Maruenda, H., Vico, M. D. L., Householder, J. E., Janovec, J. P., Cañari, C., Naka, A. y Gonzalez, A. E. (2013). Exploration of Vanilla pompona from the Peruvian Amazon as a potential source of vanilla essence: Quantification of phenolics by 
HPLC-DAD. Food Chemistry, 138(1), 161-167. https://doi. org/10.1016/j.foodchem.2012.10.037

McClements, D. J. 2004. Emulsions Ingredients. In D. J. McClements (Ed.), Food emulsions: principles, practices, and techniques (pp. 36-73). Elsevier Inc.

Monroy, J. A. (2013). Formulación de una emulsión estable de concentrado de vainilla libre de alcohol y proceso de obtención del mismo. México. (Patente MX2013014920A).

Official Methods of Analysis (1995) 16th Ed., AOAC INTERNATIONAL, Arlington, VA, sees 955.31, 964.10,966.12, $966.13,990.25$

Pérez-Silva, A., Odoux, E., Brat, P., Ribeyre, F., Rodriguez-Jimenes, G., Robles-Olvera, V., García-Alvarado, M. A. y Günata, Z. (2006). GC-MS and GC-olfactometry analysis of aroma compounds in a representative organic aroma extract from cured vanilla (Vanilla planifolia G. Jackson) beans. Food Chemistry, 99(4), 728-735. https://doi.org/10.1016/j. foodchem.2005.08.050

Qian, C. y McClements, D. J. (2011). Formation of nanoemulsions stabilized by model food-grade emulsifiers using highpressure homogenization: Factors affecting particle size. Food Hydrocolloids, 25(5), 1000-1008. https://doi. org/10.1016/j.foodhyd.2010.09.017

Salem, M. A. y Ezzat, S. M. (2019). Some New Aspects of Colloidal Systems in Foods. In J. M. Milani (Ed.), Nanoemulsions in Food Industry. IntechOpen. https://doi.org/10.5772/ intechopen.75145

Santos, J., Calero, N., Trujillo-Cayado, L. A., Martín-Piñero, M. J. y Muñoz, J. (2020). Processing and Formulation Optimization of Mandarin Essential Oil-Loaded Emulsions Developed by Microfluidization. Materials, 13(16), 3486. https://doi. org/10.3390/ma13163486

Sostaric, T., Boyce, M. C. y Spickett, E. E. (2000). Analysis of the volatile components in vanilla extracts and flavorings by solid-phase microextraction and gas chromatography. Journal of Agricultural and Food Chemistry, 48(12), 58025807. https://doi.org/10.1021/jf000515+

Tadros, T., Izquierdo, P., Esquena, J. y Solans, C. (2004). Formation and stability of nano-emulsions. Advances in Colloid and Interface Science, 108-109, 303-318. https://doi. org/10.1016/j.cis.2003.10.023

Waliszewski, K. N., Pardio, V. T. y Ovando, S. L. (2007). A simple and rapid HPLC technique for vanillin determination in alcohol extract. Food Chemistry, 101(3), 1059-1062. https:// doi.org/10.1016/j.foodchem.2006.03.004

Yalçinöz, Ş. y Erçelebi, E. (2018). Potential applications of nanoemulsions in the food systems: An update. Materials Research Express, 5(6). https://doi.org/10.1088/2053-1591/aac7ee 